\title{
NUEVA ZELANDA
}

Greta Bucher

El Colegio de México

\section{INTRODUCCIÓN}

Nueva Zelanda es un pequeño país situado en el sudoeste del océano Pacífico, conocido por sus abundantes recursos naturales, gran calidad de vida, ausencia de corrupción, buenos niveles de educación y libertades económica y políticas. Si bien es considerado como un país políticamente estable, la renuncia inesperada de John Key como primer ministro en diciembre de 2016 y los resultados de los comicios celebrados en septiembre de 2017 bajo su sucesor, Bill English, generaron cierta incertidumbre política dado que ninguna fuerza política logró conseguir la mayoría necesaria para formar gobierno de forma inmediata.

Después de 26 días de haberse celebrado los comicios, Nueva Zelanda Primero, partido político que se convirtió en kingmaker, una pieza clave para la creación del nuevo gobierno, aceptó formar una coalición con el Partido Laborista de Australia y el Partido Verde, con lo cual Jacinda Ardern, líder laborista desde agosto de 2017, se convirtió en primera ministra del país. El nuevo gobierno de coalición implicó el fin del mandato del Partido Nacional, al frente de Nueva Zelanda desde 2008.

Aunado a los retos nacionales que el nuevo gobierno laborista deberá enfrentar, no debe olvidarse el contexto internacional, marcado por la política nacionalista y aislacionista de Estados Unidos bajo el mando de Donald Trump. La nueva dirección de la política exterior estadounidense respecto a la región Asia-Pacífico podría tener ciertas implicaciones 
económicas para Nueva Zelanda. Al igual que para Australia, la posibilidad de una guerra comercial entre Estados Unidos y China - una posibilidad que si bien ha disminuido no ha desaparecido del todo - podría afectar a la economía neozelandesa debido, primero, a su relación comercial cada vez más estrecha con China (su principal socio comercial) y, segundo, a los flujos de comercio e inversión de Nueva Zelanda en la región.

De ahí que el multilateralismo se haya convertido en una opción atractiva para Nueva Zelanda. Diversificar las relaciones con otros países de la región y disminuir la dependencia económica, comercial y militar con Estados Unidos (y con China) es una de las opciones más discutidas y aceptadas tanto en Nueva Zelanda como en otras naciones. Lo anterior podría reducir el nivel de importancia de Estados Unidos en la región en términos económicos y políticos, algo que la administración previa trabajó por evitar.

En las siguientes páginas presento una evaluación de los asuntos más relevantes para Nueva Zelanda durante 2017 en materia de política nacional e internacional, económica, comercial y medioambiental, siempre considerando las posibles implicaciones que el cambio de la política exterior estadounidense, con la llegada de Donald Trump a la presidencia de Estados Unidos, pudiera tener tanto para la relación bilateral como para la realidad nacional neozelandesa. La interdependencia política, económica y militar, causa de la globalización, hace que mi estudio sea inconcluso y establezca sólo algunas pautas sobre lo que la tendencia nacionalista, aislacionista y proteccionista mundial que impera en la actualidad pueda tener para el caso neozelandés.

\section{POLÍTICA INTERNA}

Tras la sorpresiva renuncia de John Key como primer ministro el 5 de diciembre de 2016, después de 8 años de gobierno y 10 como líder del Partido Nacional, Simon William English, conocido popularmente como Bill English, fue electo como nuevo líder del Partido Nacional y como el $39^{\circ}$ primer ministro de Nueva Zelanda. Asimismo, Paula Bennett fue electa como viceprimera ministra. English, político de carrera, fue el candidato preferido de Key para sucederlo. Sin embargo, ciertos críticos no estuvieron del todo satisfechos con la decisión del liderazgo nacionalista. Además de señalar que English carece de carisma, dudaban de su 
capacidad de liderazgo ya que, en 2002, con English como su líder, el Partido Nacional sufrió su peor derrota electoral. ${ }^{1}$

En febrero de 2017, el primer ministro English anunció la celebración de elecciones generales para el 23 de septiembre del mismo año, mediante las cuales se eligió a los miembros del $52^{\circ}$ Parlamento $^{2}$ y, por ende, se formó un nuevo gobierno. En general, cada tres años se realizan elecciones generales en Nueva Zelanda. Sin embargo, por recomendación del primer ministro, la reina o el gobernador general (en su nombre) pueden disolver el Parlamento, lo que lleva a celebrar nuevos comicios. ${ }^{3}$ En este caso, Bill English recomendó la disolución del Parlamento, anticipando la celebración de elecciones por algunos meses ya que el $51^{\circ}$ Parlamento oficialmente concluía en noviembre de 2017.

Respecto a los votos obtenidos entre las principales fuerzas políticas del país, cabe mencionar que el Partido Laborista logró una gran cantidad de votos provenientes de áreas rurales, a diferencia de los nacionalistas, cuyo principal apoyo fue urbano. Asimismo, los esfuerzos del Partido Nacional de utilizar la controversial propuesta laborista de establecer un pago de regalías sobre el uso del agua a su favor no influyeron en la cantidad de votos obtenidos en las zonas rurales. De los 14 electorados en los que aumentó el voto a favor del Partido Nacional, sólo uno de ellos fue rural, el de Hunua. ${ }^{4}$ Asimismo, cabe destacar que, grosso modo, el Partido Nacional alcanzó una cantidad de votos menor en comparación con la elección de 2014 en las áreas rurales. ${ }^{5}$

1 "Bill English sworn in as New Zealand's prime minister", The Telegraph, 12 de diciembre de 2016. Consultado el 12 septiembre de 2017 en: <http://www.telegraph.co.uk/news/2016/12/11/bill-english-electedreplace-new-zealand-prime-minister-john/>.

${ }^{2}$ El Parlamento neozelandés se compone de 120 miembros, 71 electos de circunscripciones electorales y 49 por listas de partido, los cuales son electos mediante un sistema de representación proporcional mixta.

${ }^{3}$ Tras la recomendación del primer ministro, la gobernadora general, Patsy Reddy, firmó la declaración para disolver el Parlamento, la cual fue leída por el heraldo de armas extraordinario de la reina, Phillip O'Shea, durante una ceremonia pública. Una vez realizada la ceremonia, la gobernadora general redactó un decreto en el cual solicita al director general de elecciones celebrar comicios para la Cámara de Representantes. El decreto establece el último día para la nominación de candidatos, el día de la votación y el último día para que se devuelva el escrito con los nombres de los candidatos electos. Una vez celebrada la votación, la gobernadora general emite otro decreto para la formación del nuevo Parlamento dentro de las seis semanas de los resultados finales. "The official end of our Parliament (but not forever)", New Zealand Parliament, 10 de agosto de 2017. Consultado el 12 de septiembre de 2017 en: <https://www.parliament.nz/en/visit-and-learn/parliament-inelection-year/dissolution-of-parliament/the-official-end-of-our-parliament-but-not-forever>.

${ }^{4}$ Anna Bracewell-Worrall, "Digging through the data: National's urban success story", Newshub, 3 de octubre de 2017. Consultado el 11 de octubre de 2017 en: <http://www.newshub.co.nz/home/ election/2017/10/digging-through-the-data-national-s-urban-success-story.html $>$.

${ }^{5}$ Idem. 
Por otra parte, al comparar las elecciones con las de 2014, puede observarse un claro aumento de los votos obtenidos por el Partido Laborista (véase el cuadro 1). Por su parte, el Partido Nacional y sus aliados políticos —el Partido Maorí, Futuro Unido y ACT Nueva Zelanda - obtuvieron malos resultados, cada uno de ellos con menos escaños que en la elección anterior. Lo cual, además de seguir produciendo gobiernos de minoría (y por ende débiles), provocará una mayor presión para todos los partidos políticos en las siguientes elecciones dada la fragmentación del voto imperante.

Como lo venía haciendo desde 2008, el Partido Nacional esperaba conseguir la minoría necesaria (61 escaños) para formar gobierno con el apoyo de sus aliados tradicionales. Sin embargo, sólo ACT Nueva Zelanda logró conseguir una curul, lo cual dejó al Partido Nacional en una posición muy complicada, al conseguir solamente 56 escaños por su cuenta y 57 en coalición (véase el cuadro 2). Con 46 asientos, el Partido Laborista tampoco consiguió la mayoría simple. Ello generó que tanto el Partido Nacional como el Laborista entraran en negociaciones con Nueva Zelanda Primero, partido que se convirtió en el kingmaker, figura clave ya que obtuvo los escaños suficientes para formar gobierno tanto con el Partido Nacional como con el bloque Laborista-Verde. Lo anterior resultó perjudicial en especial para el Partido Nacional debido a que Nueva Zelanda Primero es considerado como un partido más afín al Partido Laborista.

Tras casi un mes de negociaciones, durante los cuales la incertidumbre política afectó al dólar neozelandés — cayó alrededor de un 3.3\% después de celebrada la elección—, ${ }^{6}$ el líder de Nueva Zelanda Primero, Winston Peters, anunció que daría su apoyo al Partido Laborista, el cual ya contaba con el favor del Partido Verde. Gracias a ello, la coalición contó con un total de 63 votos, con lo que Jacinda Ardern se convirtió en la $40^{\circ}$ primera ministra de Nueva Zelanda. Arden es la tercera mujer en ser primera ministra del país, y con 37 años de edad es la persona más joven en ocupar el cargo en los últimos 150 años. ${ }^{7}$

\footnotetext{
${ }^{6}$ Jane Wardell y Simon Cameron-Moore (eds.), "New Zealand Held in Suspense as Kingmaker Weighs Coalition Options", New York Times con información de REUTERS, 12 de octubre de 2017. Consultado el mismo día en: <https://www.nytimes.com/reuters/2017/10/12/world/asia/12reuters-newzealand-election.html>.

7 Eleanor Ainge Roy, "Jacinda Ardern to be New Zealand's next PM after Labour coalition deal”, The Guardian, 19 de octubre de 2017. Consultado el 30 de octubre de 2017 en: <https://www.theguardian. com/world/2017/oct/19/jacinda-ardern-new-zealand-prime-minister-labour-coalition-deal-winston-peters $>$.
} 
CUADRO 1. Resultados electorales por asientos totales obtenidos, 2014-2017

\begin{tabular}{|l|c|c|c|}
\hline Partido político & $\begin{array}{c}\text { Total asientos } \\
\text { obtenidos en 2014 }\end{array}$ & $\begin{array}{c}\text { Total asientos } \\
\text { obtenidos en } \\
2017\end{array}$ & $\begin{array}{c}\text { Diferencia } \\
\text { de asientos } \\
\text { obtenidos }\end{array}$ \\
\hline Partido Nacional & 60 & 56 & -4 \\
\hline Partido Laborista & 32 & 46 & +14 \\
\hline Nueva Zelanda Primero & 11 & 9 & -2 \\
\hline Partido Verde & 14 & 8 & -6 \\
\hline ACT Nueva Zelanda & 1 & 1 & -2 \\
\hline Partido Maorí & 2 & & -1 \\
\hline Futuro Unido & 1 & & \\
\hline
\end{tabular}

FUENTE: Elaboración propia con información de la Electoral Commission.

CUADRO 2. Resultados oficiales de la elección general de $2017^{8}$

\begin{tabular}{|l|c|c|c|c|c|}
\hline Partido Politico & $\begin{array}{c}\text { Votos } \\
\text { de partido }\end{array}$ & $\begin{array}{c}\% \\
\text { de votos }\end{array}$ & $\begin{array}{c}\text { Asientos } \\
\text { electorales }\end{array}$ & $\begin{array}{c}\text { Asientos } \\
\text { por lista }\end{array}$ & $\begin{array}{c}\text { Total } \\
\text { de asientos }\end{array}$ \\
\hline Partido Nacional & 1152075 & 44.4 & 41 & 15 & 56 \\
\hline Partido Laborista & 956184 & 36.9 & 29 & 17 & 96 \\
\hline Nueva Zelanda Primero & 186706 & 7.2 & - & 9 & 8 \\
\hline Partido Verde & 162443 & 6.3 & - & 8 & 1 \\
\hline ACT Nueva Zelanda & 13075 & 0.5 & 1 & - & 8 \\
\hline
\end{tabular}

FUENTE: Elaboración propia con información de la Electoral Commission.

De acuerdo con Peters, decidió apoyar al Partido Laborista porque considera que un gobierno laborista sería la mejor opción para enfrentar los retos sociales y económicos por los que atraviesa el país. No obstante, cabe destacar que existen ciertas preocupaciones por la formación de un gobierno con la participación de Nueva Zelanda Primero. Según las propias declaraciones de Peters, podría esperarse que su partido introdujera políticas

\footnotetext{
${ }^{8}$ Cabe destacar que otros partidos políticos obtuvieron votos, aunque no los necesarios para alcanzar un escaño, motivo por el cual no se incluyen en el cuadro.

${ }^{9}$ El recuento incluye los votos de los ciudadanos en el extranjero y los votos que se realizan previo a la fecha de la elección en lugares especiales para hacerlo. En el caso de esta elección, los ciudadanos pudieron emitir su voto de forma anticipada del 11 al 22 de septiembre en los lugares correspondientes. "Information for voters - the who, when and where", Electoral Commission. Consultado el 12 de septiembre de 2017 en: $<$ http://www.elections.org.nz/events/2017-general-election/information-voters-who-when-and-where>.
} 
económicas más intervencionistas, restringiera la inversión extranjera directa, redujera la migración y renegociara ciertos acuerdos comerciales.

\section{POLÍTICA EXTERIOR}

Nueva Zelanda se define como un país multilateral, imparcial e independiente que aboga por los pequeños países, en especial por aquellos que enfrentan amenazas a su paz y seguridad. Debido a ello, Nueva Zelanda está interesado en la defensa de los derechos humanos, el desarme, la no proliferación y la cooperación internacional para el desarrollo. La región AsiaPacífico es de vital importancia para el país, en particular la relación bilateral que mantiene con Australia, su principal socio comercial y aliado militar gracias al Acuerdo de Relaciones Económicas Estrechas y al Acuerdo de Relaciones de Defensa más Cercanas que ambas naciones mantienen desde 1983 y 1991, respectivamente. Asimismo, Nueva Zelanda ha desarrollado una estrategia de largo plazo para fortalecer sus vínculos en el Pacífico.

En febrero de 2017, el Ministerio de Asuntos Exteriores y Comercio publicó un documento, "The Strategic Intentions 2017-2021", en el que establece los objetivos estratégicos y las prioridades del gobierno en materia de política exterior para los siguientes cuatro años. Los objetivos incluyen la participación de Nueva Zelanda en organismos internacionales como las Naciones Unidas; aumentar el acceso a mercados, la integración económica regional y mejorar el rendimiento de las empresas neozelandesas; incorporar a Nueva Zelanda como un socio integral y confiable en la región de Asia-Pacífico; salvaguardar los intereses y la influencia de Nueva Zelanda en el Pacífico y mejorar la prosperidad, estabilidad y resiliencia de la región y de su población; promover soluciones internacionales inteligentes sobre el cambio climático, los océanos y el Antártico que aseguren la protección del medio ambiente y el uso sostenible de recursos naturales; desarrollar una capacidad de organización robusta y duradera para brindar un compromiso internacional sólido y coherente. ${ }^{10}$

10 "Strategic Intentions 2017-2021", New Zealand. Foreign Affairs and Trade, 1 de febrero de 2017. Consultado el 24 de septiembre de 2017 en: <https://www.mfat.govt.nz/assets/Uploads/MFAT-StrategicIntentions-2017-2021.pdf $>$. 
Asimismo, mediante el documento el gobierno plasmó sus prioridades para 2017 y 2018. La primera de ellas es concluir el TPP sin la participación de Estados Unidos. A la vez, también es de interés del gobierno comenzar negociaciones con la Unión Europea para crear un tratado de libre comercio; asegurar los intereses neozelandeses a medida que se implementa el Brexit; finalizar las negociaciones del tratado de libre comercio con la Alianza del Pacífico; concluir la Asociación Mejorada entre Singapur y Nueva Zelanda; alinear los esfuerzos de las agencias gubernamentales en el Pacífico; acordar una declaración actualizada de los intereses estratégicos de Nueva Zelanda en la Antártida; continuar con las negociaciones para concluir el Programa de Trabajo del Acuerdo de París a fin de que Nueva Zelanda cumpla con los mercados de carbono y sus intereses del uso de la tierra, entre otros. ${ }^{11}$

De forma simultánea, el Ministerio de Asuntos Exteriores y Comercio señala que buscará fortalecer su capacidad para gestionar las relaciones de su país con Estados Unidos y China, al tiempo que también pretende diversificar sus relaciones con el Medio Oriente, América Latina y sus socios regionales. A continuación, precisamente, se analizará la postura del gobierno neozelandés frente a Estados Unidos y China, ambos socios relevantes de Nueva Zelanda, y la propuesta existente de una mayor diversificación de su política exterior para disminuir su dependencia económica y de seguridad con ambos.

\section{Nueva Zelanda ante Estados Unidos}

Estados Unidos el tercer socio comercial de Nueva Zelanda. En 2016, el comercio bilateral sumó 16.1 mil millones de dólares. ${ }^{12}$ A la vez, es un mercado importante para la exportación de productos agrícolas y el principal destino de las exportaciones de carne de res y despojos comestibles, las cuales representaron en 2016 un valor de mil millones de dólares neozelandeses (cerca de 700 millones de dólares estadounidenses). ${ }^{13}$ Estados Unidos también es una fuente importante de inversión extranjera directa, innovación e investigación, y turismo. ${ }^{14}$ Sin embargo, como en muchos otros países, la llegada de Donald Trump a la

\footnotetext{
${ }^{11}$ Idem.
}

${ }^{12}$ Philip Matthews, "The age of uncertainty: Donald Trump, North Korea and New Zealand", Stuff, 6 de mayo de 2017. Consultado el 16 de septiembre de 2017 en: <http://www.stuff.co.nz/national/politics/ 92073664/the-age-of-uncertainty-donald-trump-north-korea-and-new-zealand $>$.

${ }^{13}$ Idem. 
presidencia de Estados Unidos generó una fuerte incertidumbre sobre el estado de la relación bilateral y su futuro. Asimismo, generó un cuestionamiento sobre la posición internacional de Nueva Zelanda y su relación, cada vez más estrecha en términos económicos, con China.

La llegada de una política exterior aislacionista y proteccionista estadounidense podría tener ciertas implicaciones para la posición internacional y el futuro de la política exterior de Nueva Zelanda. De acuerdo con un análisis elaborado por el profesor de Estudios Estratégicos de la Universidad de Victoria Robert Ayson, el aislacionismo de Estados Unidos podría ocasionar que los aliados estadounidenses — como Nueva Zelanda - replantean sus relaciones y acciones en la arena internacional. ${ }^{15}$

Ayson señala que Nueva Zelanda deberá defender los intereses neozelandeses en la relación bilateral bajo tres condiciones: (i) la inexperiencia del equipo de política exterior de Trump; (ii) la ruptura de la administración con los valores estadounidenses históricos en materia de política exterior; (iii) la idea a nivel internacional de que es posible evitar a Estados Unidos como un socio importante. ${ }^{16}$

De forma paralela, Ayson considera que motivos por los que Estados Unidos buscaba una relación más estrecha con Nueva Zelanda pueden cambiar, y el gobierno neozelandés deberá defender los valores en lo que cree y que no coinciden con los de la administración de Trump lo cual, en ciertas situaciones, generará tensiones en la relación. Nueva Zelanda tampoco puede asumir que el gobierno de Trump, mantendrá el mismo nivel de compromiso con las instituciones internacionales que ha caracterizado a Estados Unidos desde el fin de la Segunda Guerra Mundial. Sin embargo, Ayson afirma que Estados Unidos continuará siendo socio estratégico económico, militar y diplomático de Nueva Zelanda; la relación bilateral en materia de defensa no llegará a un abrupto fin y la salida de Estados Unidos a el TPP no significará un deterioro en la relación comercial. ${ }^{17}$

Por su parte, el profesor Robert G. Patman, de la Universidad de Otago, establece que la "aparente indiferencia de la administración de Trump al orden internacional liberal tiene

\footnotetext{
15 Philip Matthews, op. cit.

${ }^{16}$ Robert Ayson, “Donald Trump's Foreign Policy: Early Implications for New Zealand”, 1 de febrero de 2016. Consultado el 18 de septiembre de 2017 en: <https://www.victoria.ac.nz/hppi/centres/strategicstudies/documents/Trump-and-NZ_NZIIAtalkFeb17_Ayson.pdf $>$.

${ }^{17}$ Idem.
} 
implicaciones en la política exterior de Nueva Zelanda en términos de derechos humanos, política comercial, seguridad y en el sistema basado en reglas". ${ }^{18}$ Patman señala que la administración Trump podría complicar la relación bilateral en términos de seguridad debido a la falta de una visión estratégica coherente. ${ }^{19}$ Esto significa que la incertidumbre de la política exterior estadounidense podría afectar a Nueva Zelanda así como a todos los aliados estadounidenses. A la vez, considera que, al igual que en el caso australiano, las relaciones bilaterales de Nueva Zelanda con Estados Unidos y China podrían dificultarse. Así, el gobierno neozelandés debería diversificar sus relaciones, en especial en la región AsiaPacífico. Patman considera que impulsar el TPP sin la participación de Estados Unidos sería una manera de hacerlo.

Patman propone una política exterior de dos vías en la cual el gobierno neozelandés encuentre un balance entre la protección de intereses nacionales (la defensa de los derechos humanos, un orden internacional basado en reglas, la expansión del libre comercio) y el fortalecimiento de la cooperación en asuntos amplios de común acuerdo, como la lucha contra el Estado Islámico o el combate a la proliferación nuclear norcoreana. ${ }^{20}$ Aun cuando ambos gobiernos mantengan visiones diferentes sobre el orden internacional, Patman considera que es posible mantener relaciones cordiales al tiempo que se reconocen las diferencias políticas existentes. De forma paralela, señala que Nueva Zelanda debe mantener firme su política exterior, basada en un sistema internacional de reglas, lo que le da una voz en la arena internacional, y recordar que ambos gobiernos han sido socios estratégicos mucho antes de la llegada de Trump y lo continuarán siendo una vez que cambie la administración estadounidense.

A la vez, vale la pena mencionar que oficialmente la llegada de Trump no alteró la relación bilateral entre ambas naciones, la cual sigue definiéndose como estratégica. ${ }^{21}$ En

\footnotetext{
${ }^{18}$ Traducción propia. Robert G. Patman, "New Zealand-U.S. relations in the Trump Era and Beyond", Small States and the New Security Environment, 3 de junio de 2017. Consultado el 18 de septiembre de 2017 en: $<$ http://www.arts.canterbury.ac.nz/political/documents/ssanse2017_documents/Robert_Patman_policybrief.pdf $>$.

${ }^{19}$ Idem.

${ }^{20}$ Idem.

21 "United States of America", Foreign Affairs and Trade. New Zealand Government. Consultado el 17 de septiembre en: $<$ https://www.mfat.govt.nz/en/countries-and-regions/north-america/united-states-of-america $>$.
} 
materia de defensa, un área clave de la relación bilateral, ${ }^{22}$ el gobierno neozelandés afirma la existencia de una relación estable. Antes de ser relevado del cargo, el entonces Ministro de Defensa, Gerry Brownlee, señaló que la elección estadounidense no alteró la posición de Nueva Zelanda en temas de seguridad nacional ni a nivel regional ni global. ${ }^{23}$ Con la llegada de Mark Mitchell como ministro de Defensa, la postura de la relación bilateral se mantuvo. Mitchel, definió a Estados Unidos como "un aliado natural”, aunque resaltó la necesidad de contar estabilidad, ${ }^{24}$ algo tal vez difícil de pedir a la administración Trump.

\section{Nueva Zelanda, China y Estados Unidos}

Al igual que para Australia, una de las principales preocupaciones para el gobierno neozelandés es la posibilidad de una guerra comercial entre Estados Unidos y China. Una posibilidad que varios expertos consideran ha disminuido mas no desaparecido. La relación comercial con China, cada vez más estrecha, se ha convertido en fundamental para la economía neozelandesa. Durante la última década, la relación comercial entre ambas naciones casi se ha triplicado, pues el comercio bilateral pasando ha pasado de 8.2 mil millones de dólares en junio de 2007 a 23 mil millones de dólares en julio de $2016 .^{25}$ lo cual no significa que un desequilibro en la economía asiática pasaría desapercibido. En caso de suceder, podría tener un efecto negativo para Nueva Zelanda, ya que en la región AsiaPacífico se encuentran 6 de sus 10 principales mercados de exportación. ${ }^{26}$ Asimismo, hay que recordar que el principal socio comercial de Nueva Zelanda es Australia, país cuyo socio comercial más importante es China.

${ }^{22}$ No obstante, vale la pena mencionar que Nueva Zelanda fue suspendida del Tratado de Seguridad de Australia, Nueva Zelanda y Estados Unidos (conocido como el Tratado ANZus) en 1986, cuando declaró sus aguas territoriales como una zona libre de armas nucleares. Si bien el gobierno neozelandés reanudó áreas claves del tratado en 2007, no celebra encuentros con Australia y Estados Unidos y mantiene su zona libre de armas nucleares, mientras que Estados Unidos ha establecido una política ambigua sobre el contenido armamentístico de sus buques.

${ }^{23}$ Philip Matthews, op. cit.

${ }^{24}$ Idem.

25 "Imports and Exports", Stats NZ. New Zealand Government. Consultado el 11 de octubre de 2017 en: $<\mathrm{http}: / / \mathrm{m}$.stats.govt.nz/browse_for_stats/industry_sectors/imports_and_exports.aspx $>$.

${ }^{26}$ De acuerdo a datos compartidos por Natasha Hamilton-Hart, directora del Instituto Neozelandés de Asia de la Universidad de Auckland, durante un simposio sobre la presidencia de Donald Trump celebrado en la Universidad de Victoria en mayo de 2017. Sam Sachdeva, "How Trump vs China impacts us all", Newsroom, 9 de mayo de 2017. Consultado el 17 de septiembre de 2017 en: <https://www.newsroom. co.nz/2017/05/08/25473/trump-and-china-the-takeaways-from-the-early-days $>$. 
La diversificación de su política exterior podría ser una opción viable y necesaria para Nueva Zelanda, tal como también lo es para Australia. Mientras que académicos como Ayson no consideran que el gobierno necesariamente vaya a tener que elegir entre China y Estados Unidos (aunque podría llegar a suceder), estrechar sus relaciones comerciales, políticas y de seguridad con países como Australia o Singapur podría resultarle benéfico en el largo plazo. Asimismo, Ayson considera que el gobierno también podría acercarse a aliados menos tradicionales, como Canadá, India o Indonesia. ${ }^{27}$

De tal manera, el estatus de las relaciones bilaterales entre Nueva Zelanda y Estados Unidos y China, al menos de manera oficial, no se han alterado con la llegada de Trump a la presidencia estadounidense. Ello no exime de la existencia de un posible riesgo para los intereses neozelandeses en términos comerciales y de seguridad nacional debido a la posición incierta del presidente Trump hacia China. La situación imperante podría modificarse rápidamente, en especial debido a la amenaza en la que se ha convertido Corea de Norte para la estabilidad de la región Asia-Pacífico.

\section{ECONOMÍA}

Nueva Zelanda se define como una economía abierta y a favor del libre comercio. A lo largo de las tres últimas décadas, el gobierno reformó las regulaciones del comercio, removiendo barreras a la importación, eliminando subsidios y asegurando que las regulaciones de la inversión extranjera promovieran la llegada de inversión productiva. ${ }^{28}$ De tal manera, Nueva Zelanda pasó de ser una economía agraria, dependiente en concesiones de acceso favorables al mercado del Reino Unido, a una de libre mercado industrializada. Lo anterior incremento los ingresos reales, al tiempo que amplió las capacidades tecnológicas del sector industrial. La liberación comercial se convirtió en un factor clave para aumentar el crecimiento económico y la prosperidad de su población. Actualmente, Nueva Zelanda es la $51^{\circ}$ economía a nivel mundial con el $35^{\circ}$ PIB per cápita más alto. ${ }^{29}$

${ }^{27}$ Idem.

28 "Trade policy", Foreign Affairs and Trade. New Zealand Government. Consultado el 17 de septiembre en: <https://www.mfat.govt.nz/en/trade/nz-trade-policy/>.

29 "How New Zealand ranks", Foreign Affairs and Trade, New Zealand Government, marzo de 2017. Consultado el 26 de septiembre de 2017 en: <https://www.mfat.govt.nz/assets/Uploads/How-NZ-ranksDec2016_update-Mar2017.pdf>. 
De acuerdo con información de la Organización para la Cooperación y el Desarrollo Económico (OCDE), se espera que el crecimiento de la economía sea de alrededor de 3\% durante 2017 y $2018 .{ }^{30}$ El propio gobierno neozelandés espera que el crecimiento en 2018 sea cercano a $3.2 \%$, sin embargo pronostica una caída para 2019 y 2020 , llegando a 2.8 y $2.5 \%$, respectivamente. ${ }^{31}$ El crecimiento de 2017 y el que se espera para 2018 se apoya en la recuperación de la inversión empresarial, al nivel actual del turismo y el incremento reciente de los precios de los productos lácteos, principal producto de exportación de Nueva Zelanda.

Sin embargo, el gobierno prevé que la disminución de la migración neta y del crecimiento de los ingresos reales durante 2017 conducirán a un menor crecimiento del consumo privado, mientras que el crecimiento real del consumo público será moderado a medida que suba la inflación y disminuya el gasto público. ${ }^{32}$ Asimismo, se espera que la inflación aumente de forma constante hasta alcanzar un punto intermedio del objetivo oficial de $1-3$ por ciento. ${ }^{33}$

\section{Comercio exterior}

En aras de continuar beneficiándose del libre comercio, en marzo de 2017 el gobierno actualizó su política comercial bajo la Agenda Comercial 2030, la cual establece los objetivos en materia de comercio exterior para los siguientes 10 a 15 años. Los principales objetivos son continuar aprovechando al máximo la red de acuerdos de libre comercio con los que ya cuentan y expandirlos; enfocarse en las barreras no arancelarias al comercio exterior; aumentar su atención en los servicios, la inversión y el comercio digital; y apoyar a las empresas neozelandesas en el extranjero. ${ }^{34}$

Respecto a la actuación de la economía en materia de comercio exterior y siguiendo la información publicada por el Ministerio de Asuntos Exteriores y Comercio, para marzo de

\footnotetext{
30 "New Zealand - Economic forecast summary (June 2017)", OECD, junio de 2017. Consultado el 2 de octubre de 2017 en: <http://www.oecd.org/economy/new-zealand-economic-forecast-summary.htm>.

31 "Budget Economic and Fiscal Update 2016. Economic Outlook. Medium-term Outlook from June 2017 to 2020", The Treasury. New Zealand Government. Consultado el 2 de octubre de 2017 en: $<$ http://www.treasury.govt.nz/budget/forecasts/befu2016/008.htm>.

${ }^{32} \mathrm{Idem}$.

33 "Budget Economic and Fiscal Update 2016. Economic Outlook. Medium-term Outlook from June 2017 to 2020 ", op. cit.

34 "Trade policy", op. cit.
} 
2017 Nueva Zelanda registró un superávit comercial de 3 mil millones de dólares. El valor total de sus exportaciones de bienes y servicios aumentó $0.4 \%$ respecto al año anterior, representando un total de 70.4 mil millones de dólares, mientras que las importaciones de bienes y servicios aumentaron $0.7 \%$, lo equivalente a 67.4 mil millones de dólares (véase el cuadro 3). Los productos lácteos ${ }^{35}$ continuaron siendo los principales productos de exportación: para finales de 2017, la exportación de dichos productos registró su mayor aumento anual desde 2014, pasando de 310 millones a 13 mil millones de dólares. ${ }^{36}$ Cabe destacar, no obstante, que respecto al año anterior (marzo de 2016) el valor de las exportaciones de productos lácteos disminuyeron 0.3 por ciento. ${ }^{37}$

A la vez, para marzo de 2017 Australia continuaba siendo el socio comercial más relevante de Nueva Zelanda, así como el principal destino de las exportaciones de bienes y servicios neozelandeses. A pesar de lo anterior, vale la pena mencionar que durante los últimos cuatro años el comercio con Australia ha descendido gradualmente debido a la disminución del valor de las exportaciones de crudo, el cual ha caído $24 \%$ promedio anual desde $2013 .^{38}$

Asimismo, las exportaciones a China — segundo socio comercial de Nueva Zelanda— han aumentado en una tasa de 9\% promedio anual desde 2013. De 2016 a 2017, los principales productos de exportación fueron leña y madera y productos lácteos, ${ }^{39}$ los cuales reportaron un incremento en el volumen de exportación de 27 y de $13 \%$, respectivamente. ${ }^{40}$ En total, las exportaciones a China se mantuvieron ligeramente por debajo de los niveles máximos de 2014 (cerca de 250 mil millones de dólares). De acuerdo con el gobierno neozelandés, el crecimiento del valor de las exportaciones a China compensó el crecimiento

${ }^{35}$ Leche fresca y en polvo, mantequilla, queso, malta, caseína, albúminas, suero de mantequilla y helado.

36 "Goods and Services trade", Foreign Affair and Trade, New Zealand Government, marzo de 2017. Consultado el 26 de septiembre de 2017 en: <https://www.mfat.govt.nz/assets/Uploads/Goods-and-ServicesTrade-YE-March-2017.pdf>.

${ }^{37} \mathrm{Idem}$

${ }^{38} \mathrm{Idem}$

${ }^{39}$ Aunque durante los últimos cuatro años, el crecimiento de las exportaciones ha sido impulsado principalmente por el crecimiento de los servicios (especialmente viajes turísticos y educación), los cuales han duplicado su valor hasta llegar a 2.7 mil millones de dólares neozelandeses (cerca de 1.9 mil millones de dólares estadounidenses) en 2017. Idem.

${ }^{40}$ Idem. 
calificado como "relativamente estancado" del valor de las exportaciones con otros socios comerciales claves. ${ }^{41}$

Por otra parte, los principales orígenes de las importaciones fueron la Unión Europea (11.7 mil millones de dólares) y Australia (11.3 mil millones de dólares), siendo vehículos, maquinaria y servicios de transporte las principales importaciones. Respecto a Australia, China y Estados Unidos — tercer socio comercial de Nueva Zelanda-, los volúmenes de importaciones permanecieron constantes.

\section{Nueva Zelanda ante el Acuerdo de Asociación Transpacifico}

Como se mencionó previamente, la liberación comercial ha sido un factor clave para aumentar el crecimiento económico y la prosperidad de la población neozelandesa. Asimismo, la región Asia-Pacífico es estratégica para el crecimiento económico de Nueva Zelanda: más de $70 \%$ del comercio y la inversión del país se realiza en la región, de tal manera que el propio gobierno neozelandés afirma que el futuro del país depende de sus relaciones con los países de Asia-Pacífico. De ahí la relevancia que tenía el Acuerdo de Asociación Transpacífico para Nueva Zelanda.

Si el TPP hubiera entrado en vigor, Nueva Zelanda contaría con acceso preferencial a mercados que le son importantes y con los que aún no cuenta con acuerdos de libre comercio: Estados Unidos, Japón, Canadá, México y Perú. Nueva Zelanda exporta más de 28 mil millones de dólares en bienes y servicios a los países originales del TPP cada año, lo cual incluye a sus socios comerciales más importantes (Australia el primero; Estados Unidos el tercero; y Japón el cuarto). ${ }^{42}$ La salida de Estados Unidos del acuerdo, una de las primeras órdenes ejecutivas de Donald Trump tras asumir la presidencia en enero de 2017, fue una acción que el gobierno neozelandés lamentó.

Sin embargo, como miembro fundador del TPP y debido al interés que tiene en el mismo, el primer ministro English señaló la posibilidad de que el acuerdo continúe sin la participación estadounidense. A la vez, el entonces ministro de Asuntos Exteriores y

\footnotetext{
${ }^{41}$ Idem.

42 "Trans-Pacific Partnership Agreement", Foreign Affairs and Trade. New Zealand Government. Consultado el 16 de octubre de 2017 en: <https://www.tpp.mfat.govt.nz/about>.
} 
Comercio, Todd McClay, se reunió con sus homólogos para que el acuerdo entrara en vigor con los miembros restantes. ${ }^{43}$ Se ha planteado la posibilidad de suspender ciertas secciones del documento original, las cuales podrían ser reinstauradas en caso de que Estados Unidos regrese a formar parte del acuerdo en algún momento. ${ }^{44}$ No obstante, aún no se ha declarado nada oficial. Se espera que durante la Reunión de Líderes del APEC de noviembre de 2017 se anuncien los avances sobre el futuro del "TPP-11", es decir un TPP sin Estados Unidos.

\section{Nueva Zelanda ante la Alianza del Pacífico}

Como parte del interés del gobierno neozelandés por estrechar sus vínculos con América Latina, el 30 de junio de 2017, Nueva Zelandia inició negociaciones de libre comercio con la Alianza del Pacífico, iniciativa de integración en la que participan Chile, Colombia, Perú y México. ${ }^{45}$ Una vez concluidas las negociaciones, Nueva Zelanda se convertirá en un miembro asociado, lo que le permitirá negociar en bloque acuerdos comerciales con la Alianza. Cabe destacar que Australia, Singapur y Canadá también están en negociaciones para convertirse en miembros asociados.

De acuerdo con el entonces ministro de Comercio, Todd McClay, un acuerdo con la Alianza del Pacífico podría generar 10000 empleos y convertirse en un acceso sin precedente a los mercados latinoamericanos. ${ }^{46}$ De forma simultánea, McClay afirma que fortalecer los vínculos comerciales y empresariales con la Alianza también ayudaría a que Nueva Zelanda se convierta en un puente comercial entre América del Sur y el Sudeste Asiático. ${ }^{47}$

Dada la incertidumbre que existe sobre el futuro del TPP-11, la Alianza podría convertirse en un vínculo comercial importante para México y Nueva Zelanda, ya que no existe un tratado de libre comercio entre ambas naciones. Si bien se considera que estas dos economías son complementarias y el mercado neozelandés presenta oportunidades de

43 "Australia and New Zealand to pursue 'TPP 12 minus one'”, BBC, 24 de enero de 2017. Consultado el 16 de octubre de 2017 en: <http://www.bbc.com/news/business-38725807>.

${ }^{44}$ Idem.

${ }^{45}$ Costa Rica y Panamá son países candidatos oficiales para convertirse en miembros.

46 "McClay says jobs at the heart of Latin American trade deal", Beehire, New Zealand Government, 18 de septiembre de 2017. Consultado el 2 de octubre de 2017 en: <https://beehive.govt.nz/release/mcclay-saysjobs-heart-latin-american-trade-deal $>$.

${ }^{47}$ Idem. 
exportación para México, un incremento en la importación de lácteos neozelandeses ha generado preocupación en la industria nacional mexicana.

CuAdro 3. Principales indicadores económicos de Nueva Zelanda

\begin{tabular}{|l|l|}
\hline $\begin{array}{l}\text { PIB (precios constantes, } \\
\text { millones de dólares, junio 2017) }\end{array}$ & 268102 \\
\hline Crecimiento del PIB (2016) & $3.9 \%$ \\
\hline PIB per cápita (dólares, junio 2017) & 56482 \\
\hline $\begin{array}{l}\text { Posición en el ranking mundial de economías } \\
2017 \text { (por PIB) }\end{array}$ & 51 \\
\hline Principales exportaciones & $\begin{array}{l}\text { Productos lácteos, carne y productos cárnicos, } \\
\text { madera y productos de madera, frutas, vino, } \\
\text { maquinaria. }\end{array}$ \\
\hline Principales importaciones & $\begin{array}{l}\text { Vehículos, partes y accesorios, maquinaria y } \\
\text { equipos mecánicos, petróleo y productos derivados, } \\
\text { maquinaria y equipo eléctricos, textiles, plásticos. }\end{array}$ \\
\hline $\begin{array}{l}\text { Principales socios comerciales } \\
\text { Comercio mundial de bienes y servicios } \\
\text { (mil millones de dólares, marzo 2017) }\end{array}$ & $\begin{array}{l}\text { Australia, China, Estados Unidos y Japón } \\
\text { Exportaciones: 70.4 }\end{array}$ \\
\hline
\end{tabular}
y Stats NZ.

FUENTE: Elaboración propia con información del Banco Mundial, New Zealand Foreign Affairs and Trade

\section{MEDIO AMBIENTE}

Nueva Zelanda es un país comprometido con la preservación del medio ambiente. Al ser un país rico en recursos naturales, su gobierno trabaja para construir una economía innovadora y productiva que reduzca las emisiones de gases de efecto invernadero y sea resistente a los cambios climáticos y a los fenómenos meteorológicos adversos. ${ }^{48}$ De manera simultánea, son objetivos relevantes el cuidado y mejoramiento del agua y de la tierra, el mantenimiento sostenible de los ecosistemas marinos y la creación de ciudades sostenibles. A la vez, otro objetivo del gobierno es el desarrollo de un sistema energético mixto, el cual combine energías renovables y no renovables, ya que afirma que podría llevar a un mayor crecimiento económico y a menores emisiones en un futuro cercano. ${ }^{49}$ Durante 2017, dos temas

48 “Annual Report 2015/16”, Ministry for the Environment. New Zealand Government, octubre de 2016. Consultado el 24 de octubre de 2016 en: <http://www.mfe.govt.nz/sites/default/files/media/mfe-annual-report2016-fa.pdf>.

49 "Natural Resourses", Ministry of Business, Innovation and Employment, New Zealand Government, 9 de agosto de 2017. Consultado el 8 de octubre de 2017 en: <http://www.mbie.govt.nz/info-services/sectorsindustries/natural-resources $>$. 
medioambientales fueron notables, los cuales se abordarán a continuación: la continuación de la reforma del agua y la participación de Nueva Zelanda en el Acuerdo de París.

\section{Reforma del agua ${ }^{50}$}

Durante 2017, el gobierno neozelandés continuó trabajando en la reforma del agua, iniciada en 2009 , debido a una creciente preocupación por el deterioro de la calidad y cantidad del agua dulce, un recurso natural vital tanto en materia medioambiental como recreativa y económica. ${ }^{51}$ Tras una consulta pública realizada en febrero de $2016,{ }^{52}$ que constaba de 23 propuestas, el gobierno anunció nuevas iniciativas para continuar avanzando con la reforma del agua.

El 7 de agosto de 2017, el gobierno acordó enmendar la Declaración de Política Nacional para la Gestión del Agua Dulce de 2014. Algunas de las enmiendas, las cuales entraron en vigor el 7 de septiembre de 2017, son apoyar los objetivos nacionales del gobierno para que en 2040 se pueda nadar en 90\% de los grandes ríos y lagos del país. Para esto, se establecieron ciertos requisitos a fin de que los consejos regionales (gobiernos locales) mejoren la calidad del agua para la natación e informen cada cinco años a la población sobre sus acciones para alcanzar los objetivos regionales establecidos. ${ }^{53}$ Asimismo, los consejos regionales ahora están obligados a vigilar el progreso hacia los objetivos y

\footnotetext{
${ }^{50}$ El principal objetivo de la reforma es mejorar la administración del uso del agua, a fin de proteger el medio ambiente y lograr un crecimiento sostenible; reducir su creciente contaminación; cubrir la creciente demanda para la irrigación y la generación de energía hidroeléctrica; disminuir la sobreexplotación de los mantos acuíferos y los efectos nocivos del cambio climático; e impulsar la inversión para almacenar y distribuir dicho recurso natural. Junto con lo anterior, la reforma también aborda el reclamo histórico de los maoríes - habitantes originarios de Nueva Zelanda - quienes alegan poseer derechos históricos sobre la de propiedad del agua.

${ }^{51}$ Nueva Zelanda cuenta con más de 425000 kilómetros de ríos y arroyos, cerca de 4000 lagos y 200 mantos acuíferos. El agua es un recurso público del cual depende una gran proporción de la economía - 22\% del Producto Interno Bruto (PIB) y 67\% de las exportaciones-, ya que ésta se basa en la producción de bienes intensivos en el uso de agua: carnes, lácteos, vinos, vegetales, flores, entre otros. Además, más de la mitad de la electricidad del país se produce mediante plantas hidroeléctricas. El agua es parte fundamental de las actividades recreativas de los neozelandeses y un gran atractivo turístico. "Next steps for fresh water. Consultation document", Ministry for the Environment. New Zealand Government, febrero 2016. Consultado el 21 de marzo de 2016, en: <https://www.beehive.govt.nz/sites/all/files/1.\%20\%E2\%80\%98Next\%20Steps\% 20 for $\% 20$ Freshwater\%E2\%80\%99\%20discussion\%20document.pdf $>$.

${ }^{52}$ Idem.

53 "Latest initiatives to improve our freshwater quality", Ministry of the Environment, New Zealand Government, 9 de septiembre de 2017. Consultado el 15 de septiembre de 2017 en: <http://www.mfe. govt.nz/fresh-water/reform/latest-initiatives>.
} 
valores del agua dulce utilizando macroinvertebrados, flora y fauna autóctonas y la educación y valores maoríes; se les exige establecer métodos para responder al monitoreo y hacer públicos los resultados obtenidos; deben administrar y monitorear el nivel de nutrientes que pueden introducirse a los cuerpos de agua, como el nitrógeno y el fósforo; deben considerar el bienestar económico, ambiental, social y cultural de las comunidades en todas las etapas de la toma de decisiones, entre otras cosas. ${ }^{54}$

A la vez, como parte de las enmiendas se esclarece el significado de Te Mana o Te Wai en la administración del agua dulce. Dicho concepto reconoce al agua como un recurso natural integral al bienestar social, cultural, económico y ambiental de las comunidades, ${ }^{55}$ algo de suma importancia para los maoríes.

Asimismo, como parte de las negociaciones entre el gobierno neozelandés y los maoríes a fin de resolver disputas derivadas de interpretaciones encontradas del Tratado de Waitangi - documento fundacional de Nueva Zelanda en el cual los maoríes cedieron su soberanía al gobierno británico en 1840-, y como parte de la alianza política que ambos partidos políticos mantienen, ${ }^{56}$ en marzo de 2017 se promulgó una ley que declaró al río Whanganui, el tercero más largo del país, como una persona jurídica. ${ }^{57}$ Existirán dos guardianes que actuarán por el río, uno designado por el gobierno y otro por los maorís. Bajo el acuerdo, el gobierno también pagará una compensación por 56 millones de dólares por abusos pasados, y establecerá un fondo de 21 millones de dólares con el objetivo de mejorar la "salud y bienestar" del río. ${ }^{58}$

Es importante considerar que el gobierno de John Key (2008-2016) reconoció la existencia de derechos e intereses maoríes sobre la propiedad del agua, algo que ningún otro

54 "2017 Changes to the National Policy Statement for Freshwater Management”, Ministry of the Environment, New Zealand Government, 7 de septiembre de 2017. Consultado el 15 de septiembre de 2017 en: $<$ http://www.mfe.govt.nz/freshwater/national-policy-statement-freshwater-management/2017-changes>.

${ }^{55}$ Idem.

${ }^{56}$ A pesar de que la formación de alianzas es parte del sistema electoral neozelandés, existe la percepción de que la alianza entre el Partido Nacional y el Partido Maorí, en ocasiones fundamental para la formación del gobierno nacionalista, ha convertido al primero en cautivo de los intereses maoríes. Muriel Newman, "Politics of Fresh Water Reforms", New Zealand Center for Political Research, 28 de febrero de 2016. Consultado el 18 de marzo en: <http://www.nzcpr.com/the-politics-of-fresh-water-reform/>.

57 "New Zealand declares a river a person", The Economist, 25 de marzo de 2017. Consultado el 16 de septiembre de 2017 en: <https:/www.economist.com/news/asia/21719409-odd-legal-status-intended-helpprevent-pollution-and-other-abuses-new-zealand-declares $>$.

${ }^{58}$ Idem. 
gobierno había hecho. ${ }^{59}$ El reclamo maorí sigue en pie y se ha convertido en un tema aún más polémico. El reconocimiento del gobierno nacionalista de los derechos maoríes sobre el agua, un recurso natural definido como público en la constitución neozelandesa, y del río Whanganui como una persona jurídica, seguramente ocasionarán que el debate sobre el tema continúe.

El agua es un recurso tan importante en Nueva Zelanda que no pasó de largo durante la contienda electoral. Una de las muchas propuestas del Partido Laborista fue establecer el pago de regalías sobre el uso del agua — dependiendo de la región, la cantidad disponible y el uso que se le diera- para aquellos que sacaran beneficios comerciales de éste, con el objetivo de obtener fondos para la conservación de dicho recurso natural. ${ }^{60} \mathrm{La}$ iniciativa no fue muy bien aceptada y el Partido Nacionalista trató de utilizarla a su favor aunque, como se mencionó previamente, sin mucho éxito.

\section{Nueva Zelanda ante el Acuerdo de París}

Dado su compromiso con el medio ambiente, resultó natural la participación de Nueva Zelanda en las negociaciones del Acuerdo de París. ${ }^{61}$ El 4 de octubre de 2016 lo ratificó y presentó sus contribuciones determinadas a nivel nacional ante la Convención Marco de las Naciones Unidas sobre el Cambio Climático, las cuales pretenden reducir la emisión de gases efecto invernadero en 30\% por debajo de los niveles de 2005 para 2030. De acuerdo con el Ministerio de Medio Ambiente, con dicho objetivo se llegaría a un nivel 11\% menor que el de $1990 .{ }^{62}$ Con el propósito de alcanzar las metas planteadas, el gobierno pretende realizar reducciones de emisiones internas, eliminar dióxido de carbono mediante la expansión de bosques y participar en los mercados internacionales de carbono. Cabe resaltar que Nueva

\footnotetext{
${ }^{59}$ Muriel Newman, "Politics of Fresh Water"... op. cit.

60 "Labour's tax plan", New Zeland Labor Party. Consultado el 16 de septiembre de 2017 en: $<$ http://www.labour.org.nz/tax>.

${ }^{61}$ Si bien la mayoría de los países se sumaron a este acuerdo, no todos lo hicieron. Nicaragua y Siria, por ejemplo, no participaron en el mismo. Por otra parte, a pesar de los esfuerzos de la administración de Barack Obama por ser parte del Acuerdo de París, Donald Trump prometió durante su campaña electoral y cumplió como presidente retirar a Estados Unidos del acuerdo.

62 "The Paris Agreement", Ministry for the Environment. New Zealand Government. Consultado el 8 de octubre de 2017 en: <http://www.mfe.govt.nz/climate-change/international-forums-and-agreements/unitednations-framework-convention-climate- $0>$.
} 
Zelanda contribuye con cerca de $0.16 \%$ de las emisiones de gases de efecto invernadero a nivel mundial.

De acuerdo con un análisis elaborado por el Parlamento neozelandés, formar parte del Acuerdo de París es relevante para salvaguardar los intereses nacionales de Nueva Zelanda a largo plazo. ${ }^{63} \mathrm{Al}$ ser un país pequeño dependiente del comercio y cuyos recursos naturales y medioambientales generan un gran valor cultural, social y económico, Nueva Zelanda no puede hacer frente al cambio climático por sí solo, por lo que una acción global eficaz para combatir el cambio climático le resulta conveniente. Además, la participación neozelandesa también demuestra su compromiso con la lucha contra el cambio climático, mediante lo cual mantiene una reputación internacional positiva en la materia, algo importante para el gobierno y la sociedad de Nueva Zelanda.

Sin embargo, alcanzar los compromisos establecidos no será sencillo e implicará ciertos costos. De acuerdo con estimaciones del gobierno, alcanzar la meta de 2030 podría reducir el ingreso disponible real nacional alrededor de $1.2 \%$ anual. ${ }^{64} \mathrm{El}$ costo estimado es alto debido a que no se asumió fijar ningún precio de carbono al sector agrícola —cuyas emisiones son relativamente altas a nivel internacional, al representar $49 \%$ del total de las emisiones del país-, el poco desarrollo tecnológico y el ritmo de crecimiento forestal. No obstante, el costo estimado podría modificarse dependiendo de las políticas nacionales que se implementen en los próximos años. De forma paralela, Nueva Zelanda enfrentará mayores costos en comparación con otros países desarrollados debido a que, actualmente, más de $80 \%$ de la electricidad del país ya es renovable. ${ }^{65}$

A pesar de lo anterior, vale la pena mencionar que el gobierno neozelandés considera que el acuerdo también representa oportunidades económicas para Nueva Zelanda. Gracias a sus abundantes recursos naturales y mediante la innovación, Nueva Zelanda puede generar nuevos empleos. Asimismo, puede defender su reputación a nivel internacional en áreas como el comercio y la política exteriores.

63 "National Interest Analysis: The Paris Agreement", New Zealand Parliament, 17 de agosto de 2016. Consultado el 9 de octubre de 2017 en: <https://www.parliament.nz/resource/en-NZ/51DBHOH_PAP69732_ 1/b3d874584455ca4c02251f71995b03d6f0aeee42>.

${ }^{64} \mathrm{Idem}$.

${ }^{65}$ Idem. 
Respecto a la decisión del presidente Donald Trump de retirar a Estados Unidos —el segundo emisor de gases invernaderos más grande del mundo- del Acuerdo de París en junio de 2017, Nueva Zelanda, junto con países como Francia, Japón y Australia, reiteraron su determinación para afrontar el cambio climática mediante el acuerdo y otras iniciativas. La entonces primera viceministra Paula Bennett confirmó que Nueva Zelanda está comprometido a "jugar su parte para afrontar el cambio climático global". ${ }^{66}$

Por otra parte, un sector de la sociedad neozelandesa manifestó de forma muy clara su decepción por la salida de Estados Unidos del Acuerdo. Durante la visita del secretario de Estado estadounidense Rex Tillerson, la cual se llevó a cabo en junio de 2017, un grupo de manifestantes lo recibieron con señas obscenas. ${ }^{67} \mathrm{Si}$ bien Tillerson trató de asegurar durante una conferencia de prensa que la salida de Estados Unidos del Acuerdo de París no significa que dicho país dejará de trabajar para reducir la emisión de gases de efecto invernadero, su explicación fue poco convincente. ${ }^{68}$

${ }^{66}$ Stacey Kirk, "The Paris Agreement - is New Zealand as committed without the US?", Stuff, 2 de junio de 2017. Consultado el 8 de octubre de 2017 en: <http://www.stuff.co.nz/national/politics/93216195/the-parisagreement--is-new-zealand-as-committed-without-the-us $>$.

${ }^{67}$ Matt Novak, "Trump Regime Greeted by Middle Fingers in New Zealand After Dumping Climate Accord", Gizmodo, 6 de junio de 2017. Consultado el 9 de octubre de 2017 en: <https://gizmodo.com/trumpregime-greeted-by-middle-fingers-in-new-zealand-a-1795842539>.

${ }^{68}$ Idem. 


\section{APÉNDICE}

\begin{tabular}{ll}
\hline Nombre oficial & Nueva Zelanda o Nueva Zelandia \\
Capital & Wellington \\
Extensión territorial $\left(\mathrm{km}^{2}\right)$ & 267710.0 \\
Población (octubre de 2017) & 4821881 \\
Religión & No existe religión de Estado \\
Idiomas & El inglés, el maorí y el lenguaje de señas de Nueva Zelanda \\
Moneda & Dólar neozelandés \\
Gobierno & Democracia parlamentaria \\
Jefe de Estado & La reina Isabel II, cuyo representante es la gobernadora general \\
& Patsy Reddy desde el 28 de septiembre de 2016 \\
Jefe de Gobierno & La primera ministra Jacinda Ardern desde el 26 de octubre de \\
& 2017. Su predecesor: Bill English. \\
Principales organizaciones políticas & Partido Nacional, Partido Laborista, Partido Maorí, ACT Nueva \\
& Zelanda, Partido Verde, Nueva Zelanda Primero, Futuro Unido
\end{tabular}

Ministros clave:

Primer viceministro

Finanzas

Asuntos Internacionales

Defensa

Medio Ambiente

Asuntos Maorís

Justicia

Fiscal general
Winston Peters. Predecesora: Paula Bennett

Grant Robertson. Predecesor: Steven Joyce

Winston Peters. Predecesor: Gerry Brownlee

Ron Mark. Predecesor: Mark Mitchell

David Parker. Predecesor: Nick Smith

Nanaia Mahuta. Predecesor: Te Ururoa Flavell

Andrew Little. Predecesor: Amy Adams

David Parker. Predecesor: Chris Finlayson

FUENTE: Elaboración propia con información del Banco Mundial, Foreign Affairs and Trade New Zealand Government, Stats New Zealand Government y CIA World Factbook. 


\section{BIBLIOGRAFÍA}

Banco Mundial, “GDP ranking”, 1 de julio de 2017. Consultado el 29 de agosto de 2017 en: $<$ https://data.worldbank.org/data-catalog/GDP-ranking-table $>$.

BBC, "Australia and New Zealand to pursue 'TPP 12 minus one"”, BBC, 24 de enero de 2017. Consultado el 16 de octubre de 2017 en: <http://www.bbc.com/news/business$3872580>$.

Beevihe New Zealand Government, "McClay says jobs at the heart of Latin American trade deal", 18 de septiembre de 2017. Consultado el 2 de octubre de 2017 en: $<$ https://beehive.govt.nz/release/mcclay-says-jobs-heart-latin-american-trade-deal $>$.

"Donald Trump's Foreign Policy: Early Implications for New Zealand", Robert Ayson, 1 de febrero de 2016. Consultado el 18 de septiembre de 2017 en: <https://www. victoria.ac.nz/hppi/centres/strategic-studies/documents/Trump-and-NZ_NZIIAtalk Feb17_Ayson.pdf>.

Electoral Commission, "2017 General Election - Official Results". Consultado el 10 de octubre de 2016 en: <http://www.electionresults.govt.nz/electionresults_2017/>.

Electoral Commission, "Information for voters - the who, when and where". Consultado el 12 de septiembre de 2017 en: <http://www.elections.org.nz/events/2017-generalelection/information-voters-who-when-and-where>.

Foreign Affairs and Trade. New Zealand Government, "Goods and Services trade", marzo 2017. Consultado el 26 de septiembre de 2017 en: <https://www.mfat.govt.nz/assets/ Uploads/Goods-and-Services-Trade-YE-March-2017.pdf $>$.

Foreign Affairs and Trade. New Zealand Government, "How New Zealand ranks", marzo de 2017. Consultado el 26 de septiembre de 2017 en: <https://www.mfat.govt.nz/ assets/Uploads/How-NZ-ranks-Dec2016_update-Mar2017.pdf>.

Foreign Affairs and Trade. New Zealand Government, "Strategic Intentions 2017-2021", 1 de febrero de 2017. Consultado el 24 de septiembre de 2017 en: $<$ https://www.mfat. govt.nz/assets/Uploads/MFAT-Strategic-Intentions-2017-2021.pdf $>$. 
Foreign Affairs and Trade. New Zealand Government, "Trade policy". Consultado el 17 de septiembre en: <https://www.mfat.govt.nz/en/trade/nz-trade-policy/>.

Foreign Affairs and Trade. New Zealand Government, "Trans-Pacific Partnership". Consultado el 16 de octubre de 2017 en: <https://www.tpp.mfat.govt.nz/about>.

Foreign Affairs and Trade. New Zealand Government. "United States of America". Consultado el 17 de septiembre en: <https://www.mfat.govt.nz/en/countries-andregions/north-america/united-states-of-america> .

Gizmodo, Matt Novak, "Trump Regime Greeted by Middle Fingers in New Zealand after Dumping Climate Accord", , 6 de junio de 2017. Consultado el 9 de octubre de 2017 en: $<$ https://gizmodo.com/trump-regime-greeted-by-middle-fingers-in-new-zealand-a$1795842539>$.

Labor Party, "Labour's tax plan". Consultado el 16 de septiembre de 2017 en: $<$ http://www.labour.org.nz/tax $>$.

Ministry of Business, Innovation and Employment, New Zealand Government, "Natural Resourses", 9 de agosto de 2017. Consultado el 8 de octubre de 2017 en: $<$ http://www.mbie.govt.nz/info-services/sectors-industries/natural-resources $>$.

Ministry for the Environment, New Zealand Government, "2017 changes to the National Policy Statement for Freshwater Management", 7 de septiembre de 2017. Consultado el 15 de septiembre de 2017 en: <http://www.mfe.govt.nz/freshwater/national-policystatement-freshwater-management/2017-changes $>$.

Ministry for the Environment, New Zealand Government, “Annual Report 2015/16”, octubre 2016. Consultado el 24 de octubre de 2016 en: <http://www.mfe.govt.nz/sites/ default/files/media/mfe-annual-report-2016-fa.pdfs.

Ministry for the Environment, New Zealand Government, "Latest initiatives to improve our freshwater quality", 9 de septiembre de 2017. Consultado el 15 de septiembre de 2017 en: <http://www.mfe.govt.nz/fresh-water/reform/latest-initiatives $>$.

Ministry for the Environment, New Zealand Government, "Next steps for fresh water. Consultation document", febrero 2016. Consultado el 21 de marzo de 2016, en: 
$<$ https://www.beehive.govt.nz/sites/all/files/1.\%20\%E2\%80\%98Next\%20Steps\%20f or\%20Freshwater\%E2\%80\%99\%20discussion\%20document.pdf>.

Ministry for the Environment, New Zealand Government, "The Paris Agreement", Consultado el 8 de octubre de 2017 en: <http://www.mfe.govt.nz/climate-change/ international-forums-and-agreements/united-nations-framework-convention-climate- $0>$.

Newshub, Anna Bracewell-Worrall, "Digging through the data: National's urban success story", 3 de octubre de 2017. Consultado el 11 de octubre de 2017 en: $<$ http://www.newshub.co.nz/home/election/2017/10/digging-through-the-data-nationals-urban-success-story.html $>$.

Newshub, Patrick Gower, "Bill English bags Donald Trump's chaotic White House”, 20 de mayo de 2017. Consultado el 17 de septiembre de 2017 en: <http://www.newshub.co.nz/ home/politics/2017/05/bill-english-bags-donald-trump-s-chaotic-white-house.html>.

New York Times, (eds.) Jane Wardell y Simon Cameron-Moore, "New Zealand Held in Suspense as Kingmaker Weighs Coalition Options", con información de REUTERS, 12 de octubre de 2017. Consultado el mismo día en: < https://www.nytimes. com/reuters/2017/10/12/world/asia/12reuters-newzealand-election.html $>$.

New Zealand Center for Political Research, Muriel Newman, "Politics of Fresh Water Reforms", 28 de febrero de 2016. Consultado el 18 de marzo, en: <http://www. nzcpr.com/the-politics-of-fresh-water-reform/>.

New Zealand Parliament, "National Interest Analysis: The Paris Agreement", 17 de agosto de 2016. Consultado el 9 de octubre de 2017 en: <https://www.parliament.nz/ resource/en-NZ/51DBHOH_PAP69732_1/b3d874584455ca4c02251f71995b03d6f0 aeee $42>$.

New Zealand Parliament, "The official end of our Parliament (but not forever)", 10 de agosto de 2017. Consultado el 12 de septiembre de 2017 en: <https://www.parliament.nz/ en/visit-and-learn/parliament-in-election-year/dissolution-of-parliament/the-officialend-of-our-parliament-but-not-forever/>. 
Newsroom, Sam Sachdeva, "How Trump vs China impacts us all", 9 de mayo de 2017. Consultado el 17 de septiembre de 2017 en: <https://www.newsroom.co.nz/2017/ 05/08/25473/trump-and-china-the-takeaways-from-the-early-days $>$.

OECD, "New Zealand-Economic forecast summary (June 2017)", $O E C D$, junio de 2017. Consultado el 2 de octubre de 2017 en: <http://www.oecd.org/economy/new-zealandeconomic-forecast-summary.htm>.

Small States and the New Security Enironment, Robert G. Patman, "New Zealand-U.S. relations in the Trump Era and Beyond", 3 de junio de 2017. Consultado el 18 de septiembre de 2017 en: <http://www.arts.canterbury.ac.nz/political/documents/ ssanse2017_documents/Robert_Patman_policybrief.pdf $>$.

Stats NZ, New Zealand Government, "Imports and Exports". Consultado el 11 de octubre de 2017 en: <http://m.stats.govt.nz/browse_for_stats/industry_sectors/imports_and exports.aspx $>$.

Stats NZ, "Top Statistics", junio de 2017. Consultado el 3 de octubre de 2017 en: $<$ http://www.stats.govt.nz/browse_for_stats/snapshots-of-nz/top-statistics.aspx $>$.

Stuff, "US President Donald Trump speaks with Prime Minister Bill English", 7 de febrero de 2017. Consultado el 16 de septiembre de 2017 en: < http://www.stuff.co.nz/national/ politics/89122833/US-President-Donald-Trump-speaks-with-Prime-Minister-BillEnglish>.

Stuff, Laura Mcquillan, "Prime Minister Bill English to speak with US President Donald Trump for the first time", 6 de febrero de 2017. Consultado el 16 de septiembre de 2017 en: $<$ http://www.stuff.co.nz/national/politics/89117434/Prime-Minister-Bill-English-to-speakwith-US-President-Donald-Trump-for-the-first-time> .

Stuff, Stacey Kirk, "The Paris Agreement - is New Zealand as committed without the US?", 2 de junio de 2017. Consultado el 8 de octubre de 2017 en: $<$ http://www.stuff.co.nz/national/ politics/93216195/the-paris-agreement--is-new-zealand-as-committed-without-the-us $>$.

Stuff, Philip Mathews, "The age of uncertainty: Donald Trump, North Korea and New Zealand", 6 de mayo de 2017. Consultado el 16 de septiembre de 2017 en: 
$<$ http://www.stuff.co.nz/national/politics/92073664/the-age-of-uncertainty-donaldtrump-north-korea-and-new-zealand $>$.

The Economist, "New Zealand declares a river a person", 25 de marzo de 2017. Consultado el 16 de septiembre de 2017 en: <https://www.economist.com/news/asia/21719409-oddlegal-status-intended-help-prevent-pollution-and-other-abuses-new-zealand-declares $>$.

The Guardian, Eleanor Ainge Roy, "Jacinda Ardern to be New Zealand's next PM after Labour coalition deal", 19 de octubre de 2017. Consultado el 30 de octubre de 2017 en: $<$ https://www.theguardian.com/world/2017/oct/19/jacinda-ardern-new-zealand-primeminister-labour-coalition-deal-winston-peters $>$.

The Telegraph, "Bill English sworn in as New Zealand's prime minister", 12 de diciembre de 2016. Consultado el 12 septiembre de 2017 en: <http://www.telegraph.co.uk/news/ 2016/12/11/bill-english-elected-replace-new-zealand-prime-minister-john/>.

The Treasury. New Zealand Government, "Budget Economic and Fiscal Update 2016. Economic Outlook. Medium-term Outlook from June 2017 to 2020". Consultado el 2 de octubre de 2017 en: <http://www.treasury.govt.nz/budget/forecasts/befu2016/008.htm>. 